\title{
AULAS UNIVERSITARIAS, TECNOLOGÍAS DIGITALES Y CULTURA DE LA PARTICIPACIÓN
}

\author{
University classrooms, digital technologies and participation culture?
}

Ileana Cruz Sánchez y Luz María Garay

Fecha de recepción: 30/06/18

Fecha de aceptación: 13/12/18

RESUMEN: El artículo presenta resultados de una investigación cuyo objetivo fue analizar cómo se promueve la cultura de la participación en aulas universitarias mexicanas, se elaboraron cuatro estudios de caso correspondientes a cuatro docentes que han incoporado las tecnologías y entornos digitales en sus prácticas de enseñanza. Se realizaron entrevistas a profundidad y observación no participante durante un semestre en los escenarios físicos y los entornos digitales que usan para sus clases y se llevaron a cabo grupos de discusión con los estudiantes para analizar su percepción sobre el uso de tecnología digital en su proceso de aprendizaje. Se tomó como punto de partida el concepto de cultura participativa de Henry Jenkys, se elaboró un recorrido conceptual en relación con la inserción de las tecnologías digitales en la educación, los actores del proceso y las concepciones de aprendizaje de los docentes para comprender las decisiones al elegir tecnologías digitales y diseñar actividades de aprendizaje. Se realizó un análisis de las distintas prácticas de uso de las tecnologías que promueven los docentes a partir del concepto de cultura participativa de Jenkins, finalmente se presenta una discusión que nos permité analizar qué tanto se ha logrado promover la cultura participativa en esas aulas universitarias.

PALABRAS CLAVE: educación, tecnologías de la información, participación, docentes.

ABSTRACT: The article presents the results of a research initiative that analyses how the culture of participation in Mexican university classrooms is promoted. To that aim, four case studies corresponding to four teachers who have incorporated digital technologies and environments into their teaching practices were developed. In-depth interviews and nonparticipant observations were conducted during a semester in the physical scenarios and digital environments they use for their classes. Simultaneously, discussion groups were held with students for examining their perception of the use of digital technology in their learning process. The concept of the participative culture of Henry Jenkins [year] was taken as a starting point. Aa conceptual journey in relation to the insertion of digital technologies in education was elaborated, in which the actors of the process and to teachers' learning conceptions to understand the decisions that lead them to choose digital technologies and design learning activities. Also, based on Jenkins' concept of participatory culture, an analysis of the different practices of the use of technologies promoted by teachers was made. Finally, a discussion was presented to measure the extent in which participatory culture has been promoted in these university classrooms.

KEY WORDS: education, information technologies, participation, teachers 


\section{Introducción}

En los salones de las instituciones de educación superior mexicanas, cada vez es más frecuente ver computadoras, exposiciones auxiliadas por un proyector y alguna presentación digital, o bien escuchar a los maestros que solicitan los trabajos vía correo electrónico o a través de la plataforma virtual que se emplee en la universidad. Al mismo tiempo, se adoptan metodologías que incorporan herramientas tecnológicas como auxiliares a los procesos educativos. Profesores y estudiantes se comunican a través de plataformas, conforman grupos en distintas redes sociodigitales, utilizan los mensajes personales. Las instituciones invierten recursos en dotar a sus instalaciones de conectividad mediante redes wifi y salas de cómputo, servidores y otros recursos tecnológicos.

Los universos digitales permiten y fomentan una cultura participativa que puede naturalizarse en las aulas -presenciales y digitales- a través de la acción docente, de la apropiación y adaptación de las tecnologías digitales como herramientas de la mente y con ello potenciar las habilidades y competencias de sus estudiantes. Este texto da cuenta de algunos de esos procesos de apropiación y uso, observa los cómo y los para qué en ese objeto de estudio ${ }^{1}$.

La cultura participativa se define como una nueva forma de interactuar en la cual los usuarios o participantes tienen la posibilidad de crear, remezclar, compartir y curar contenidos -principal, aunque no exclusivamente- en diversos escenarios digitales. Más adelante se profundizará en el concepto a partir de la propuesta realizada por Jenkins.

¿Qué se sabe de la cultura de la participación en ámbitos educativos en México? ¿Qué se ha trabajado con la cultura participativa en las aulas mexicanas? Es un tema incipiente aún, ya que cultura participativa se relaciona más con la participación ciudadana, específicamente con la participación ciudadana electoral, que con la educación; así que a partir de esas preguntas se desprende el objetivo de este artículo: analizar la cultura de la participación y sus actores en cuatro contextos áulicos universitarios para tratar de analizar y comprender este fenómeno emergente en ambientes escolares.

\section{Escenarios de investigación, categorías de análisis y estrategia metodológica}

La perspectiva que se siguió en esta investigación se inscribe en el campo de la comunicación-educación, y sigue la tradición de la escuela canadiense en su aplicación de tecnología educativa, puesto que propone una aplicación metodológica para las narrativas transmedia en el aula; en relación con la cultura digital sigue los pasos de Henry Jenkins al centrarse en la cultura participativa y las narrativas transmedia.

Se combinó el trabajo de orden documental y el uso de métodos interpretativos como 1) entrevistas a profundidad, 2) observación no participante, 3) grupos de discusión, 4) análisis de entornos digitales; cada uno de estos momentos fueron realizados en etapas que presuponían, por su parte, recursos y procedimientos específicos (Álvarez-Gayou, 2012).

El trabajo de campo se llevó a cabo durante dos semestres lectivos, dado que fue indispensable observar las interacciones de los participantes en los ambientes áulicos y digitales. Los semestres en los cuales se llevaron a cabo las observaciones, entrevistas y

\footnotetext{
${ }^{1}$ Este artículo se desprende de una investigación mayor, la tesis doctoral "Cultura de la participación y aprendizaje digital. Hacia una propuesta de diseño educativo transmedial".
} 
grupos de discusión fueron el 2015-2 (agosto-diciembre) y el 2016-1 (enero-junio); las observaciones y análisis de los entornos digitales se llevaron a cabo al finalizar cada uno de los semestres para no causar incomodidad en los estudiantes y no interferir en el proceso de evaluación y calificación de los estudiantes en los entornos digitales.

El constructo o variable fundamental es la cultura de la participación, que se construye a partir de entornos, contextos y usuarios o actores. Por tanto, se investigaron las características de dichos actores -que al interior de un aula son docentes y estudiantes- y sus formas de participación en la creación de contenidos digitales académicos en los escenarios áulicos.

Para observar lo que sucedía en el interior de las aulas con la cultura participativa, se construyeron tres grandes categorías de análisis:

a) Escenarios de participación: las aulas y los espacios digitales en donde se llevan a cabo las interacciones académicas entre docentes y estudiantes.

b) Actantes, en donde se analizan las características de docentes, estudiantes y dispositivos y las relaciones entre ellos. El nombre de la categoría se desprende la teoría del actor-red de Bruno Latour (1996).

c) Cultura participativa, a través de la cual se analizan los tipos de participación de acuerdo con la propuesta de Jenkins (2009) afiliación, circulación, expresión y trabajo colaborativo en los distintos escenarios para encontrar rasgos comunes que complementen a las dos categorías anteriores.

El estudio contempló tres fases que permitieron comprender mejor la complejidad del fenómeno: La primera fase fue diseñada para conocer a los informantes docentes y su entorno de trabajo: el aula. La segunda fase se dedicó a conocer a los estudiantes. Las dos primeras fases se realizaron simultáneamente dado que en la observación se trabaja tanto con estudiantes como con docentes; la realización de los grupos de discusión se llevó a cabo al término de la observación. La tercera fase estuvo diseñada para observar la participación de los actores en las distintas aplicaciones digitales y servicios que se utilizan para el proceso enseñanza-aprendizaje y analizar los contenidos (o productos) académicos digitales.

Se trabajó con cuatro docentes voluntarios, en este artículo se presentan resultados de los cuatro pero se detallan los hallazgos del caso del profesor Miguel; esta decisión se tomó considerando tres factores: a) la peculiar formación que tiene el docente con el que se trabajó pues ha desarrollado dos áreas de conocimiento que se complementan de manera interesantes. El docente cuenta con los siguientes estudios: Licenciatura en Música con especialidad en Educación Musical, Ingeniería Industrial y de Sistemas, Ingeniería de Audio, Maestrante en Educación Superior, Maestría en Administración y Candidatura a Doctor en Administración, b) El tipo de asignaturas impartidas en dos áreas y dos carreras diferentes, y c) se realizó una observación más sistemática durante un semestre lectivo a diferencia de los otros informantes.

\subsection{Escenarios de participación, instituciones educativas presenciales}

Para el estudio general se seleccionaron dos instituciones públicas: la Universidad Autónoma de Querétaro y el Tecnológico Nacional de México, campus Ocotlán ${ }^{2}$, fundado en

\footnotetext{
${ }^{2}$ El el momento del trabajo de campo era el Instituto Tecnológico de Ocotlán. La denominación oficial cambió a partir de 2018.
} 
1991 en la región de la Ciénega del Estado de Jalisco, dependiente de la Secretaría de Educación Pública.

Y dos instituciones privadas: la Universidad del Valle de México, campus Querétaro y la Universidad Anáhuac Querétaro.

\section{Escenarios institucionales presenciales del estudio de caso presentado}

La Universidad Anáhuac Querétaro es relativamente pequeña. Cuenta con tres edificios y grandes áreas abiertas. Los estudiantes y trabajadores pueden conectarse a internet mediante la red wifi al identificarse con su clave, sin embargo, la conexión a veces falla.

La observación se realizó en el semestre 2015-2 en dos asignaturas: Creación y producción musical de la Licenciatura en Dirección de Empresas del Entretenimiento y, Cálculo, asignatura de tronco común de las licenciaturas del área de negocios. Los grupos observados eran pequeños: cuatro estudiantes en Creación y producción musical y doce en Cálculo.

El escenario en la Anáhuac Querétaro es un salón con una distribución casi isóptica, con desniveles escalonados para permitirle a los estudiantes ver mejor al maestro, al pintarrón o la pantalla. En cada nivel, tres mesas juntas, a modo de hilera, y tres o cuatro sillas para cada mesa. Al fondo una mesa hace las veces de escritorio para el maestro.

Los salones de la Anáhuac Querétaro se prestan para que el profesor imparta cátedra y los estudiantes se sienten en líneas; el mobiliario dificulta la formación de trabajo colaborativo dentro del aula. Poseen las facilidades tecnológicas como conexión para diferentes tipos de computadora, cañón y pantallas para presentaciones, que el docenteinformador utiliza como apoyos.

\subsection{Escenarios de participación digital. Facebook, Google Drive}

De acuerdo con Cobo Romaní y Pardo Kuklinski, Facebook es una red social con alto sentido de apropiación y pertenencia de los usuarios, constituyéndose como un "espacio para formar relaciones, comunidades, y otros sistemas sociales donde rigen normas similares a las del mundo real, en donde la participación está motivada por la participación” (2007, p. 64).

Tres de los informantes-docentes que participaron en la investigación reportaron en las entrevistas utilizar Facebook como herramienta digital, a través de la creación de un grupo de la asignatura. Su uso en todos los casos fue para compartir información, cuestiones de gestión administrativa (avisos, solicitud de permisos) y en uno de los casos, para resolver dudas. Sólo se tuvo acceso a observar dos grupos de clase en esta red social, en donde hubo trescientas publicaciones, de las cuales 42 fueron vínculos a archivos creados por el docente, ya sean presentaciones de apoyo para la clase, problemarios o rúbricas de evaluación; 208 publicaciones -tanto por parte del docente como de los estudiantes-correspondieron a videos que apoyaban los temas de la clase o bien videos para analizar las piezas musicales que eran tema de la asignatura, avisos, memes, fotografías, invitaciones, links a libros, software, información complementaria, invitaciones a eventos; y 110 corresponden a comentarios en las publicaciones.

Google Drive., este servicio o mashup -en la clasificación de Cobo Romaní y Pardo Kuklinski (2007)- ofrece además del almacenamiento de archivos, software de ofimática 
nativo (procesador de texto, hoja de cálculo, editor de formularios, presentaciones y visor de PDF), que sincroniza los cambios en la nube.

Dentro de sus funciones permite compartir archivos y carpetas con otros usuarios, el trabajo simultáneo de varios usuarios en el mismo documento, así como el uso de comentarios y otro tipo de notas para facilitar la edición conjunta. Además, cuenta con una herramienta de escritorio a través de la cual es posible trabajar offline (Wikipedia, 2017).

Dos de los informantes-docentes utilizan esta herramienta como repositorio de archivos que comparten con sus estudiantes, así como para revisar los trabajos que les solicitan. Uno de ellos reporta el uso de la herramienta en el tiempo de la clase, lo que les permite a los estudiantes hacer las correcciones de forma síncrona y asíncrona. En este servicio se observaron dos grupos de dos profesores, los cuales crearon 11 contenidos originales: 7 presentaciones electrónicas, 3 textos y una hoja electrónica. Los estudiantes colaboraron con 50 podcast, algunos de los cuales son correcciones al mismo trabajo, 26 documentos, 4 matrices y dos cómics.

Los escenarios digitales seleccionados por los docentes informantes son parte del entorno tecno-social Web 2.0, y como tales cumplen con las características enumeradas por Fumero, Roca y Sáez Vacas (2007) de representación, captación y manejo de la información, modificaciones sensoriales, relacionales y en la identidad mediante el uso de avatares, son funcionales en su infraestructura sociotécnica y permiten modificaciones espaciotemporales. Una de las principales razones para utilizarlas es que sólo requieren un registro de usuario para su utilización, son gratuitas.

Los informantes docentes utilizan, además de las observadas, una amplia variedad de aplicaciones y servicios. Los seleccionan basados en su aplicabilidad, porque potencian su creatividad o porque facilitan el trabajo cotidiano de los estudiantes.

\section{Actantes estudiantes y docentes. Uso de los entornos digitales, dispositivos}

Los dispositivos con los que interactuaban tanto estudiantes como docentes fueron laptops, teléfonos inteligentes y tabletas. En algunas de las clases se utilizaban para el trabajo de clase, sea individual o colectivo; en otras los estudiantes tomaban notas, buscaban información.

En las cuatro instituciones de educación superior se observó la presencia de al menos un dispositivo por estudiante, a veces más. Los informantes docentes perciben a las tecnologías digitales como aliadas, como algo con lo que sus estudiantes están familiarizados y promueven su uso dentro de las aulas.

A través de las distintas técnicas de investigación, se corroboró que la presencia de los dispositivos móviles en la clase, como en otros escenarios de la vida de los participantes, es algo cotidiano. A decir de los informantes, se tiene literalmente a la mano. Y este uso transparente de los dispositivos, otorga a los estudiantes autonomía y control sobre su aprendizaje, al tiempo que refuerza sus habilidades digitales.

Los estudiantes perciben el uso de los medios digitales como facilitadores debido a la ubicuidad, como puentes de la relación entre docentes y estudiantes y también como una tendencia de esta época. 
Las tecnologías digitales son utilizadas para potenciar el aprendizaje de los estudiantes o para resolver dudas sin exponerse al escrutinio del resto del grupo. Los dispositivos se convierten en un puente entre las personas, no solo un medio para hacerse de información o como auxiliar en alguna asignatura.

Siempre, como lo que quiero es que los chavos aprendan de cómo aprenden, hago grupos de Facebook, [...] lo que yo veo es que comparten muchas cosas y cuando menos los invita a que en privado, porque siempre me terminan mandando algún chat cuando quieren preguntar algo, sirve para tener el acceso hacia alguien sin que los demás se enteren. Por alguna razón, nos sigue dando miedo que los demás sepan o se enteren de que yo no entendí algo (Miguel).

Además de los grupos en Facebook, Miguel utilizó el Google Drive para que los estudiantes realizaran sus trabajos. Se observó que, en algunas de las clases de Producción de audio, los avances los realizaban en el tiempo de clase, lo que permitía al maestro corregir o hacer comentarios sobre lo que escribían. Al respecto, en el grupo de discusión las estudiantes comentaron

Cada uno tenía una carpeta y estábamos todos trabajando en ella y entonces el profesor, mientras nosotros íbamos cambiando cosas, iba viendo ahí, y te iba poniendo comentarios '¿segura que quieres decir esto?' y así (Kathy AnáhuacQro).

Otra forma en que se utilizan las tecnologías digitales y los dispositivos es como repositorios de los materiales de la asignatura, pero también como medios de control, puesto que las plataformas digitales permiten a los creadores de los grupos de Facebook o de las asignaturas en el caso de las plataformas de manejo de contenidos, visualizar quién vio los recursos publicados o el tipo de actividad de los usuarios, la hora y el tiempo de conexión.

Los dispositivos son actantes que permiten la interacción entre los otros actantes y con los materiales que se comparten. Además, se transforman en espacios de trabajo síncrono o asíncrono, así como en una forma privada de resolver dudas. Su uso, si bien permite la edición síncrona de documentos -en el caso de GoogleDrive-, puede sentirse una intrusión a los ritmos de cada individuo. "Yo me sentía súper espiada" (Maruja Anáhuac-Qro), "es como cuando estás haciendo un examen y cuando se para junto a ti el profesor" (Kathy AnáhuacQro). Es necesario, entonces buscar acuerdos en la forma de trabajo síncrona, porque el docente no es considerado como par, y su intervención puede resultar incómoda.

Los dispositivos son aliados al alcance de la mano, y los estudiantes consideran importante utilizarlos de acuerdo con el contexto en el que se está, en este caso, el académico:

Creo que, a pesar de tener la tecnología a la mano, somos bastante conscientes de que las herramientas que estás utilizando para la clase. Somos lo suficientemente maduros para ocupar la computadora en la clase (Maruja AnáhuacQro).

Uno de los usos de los escenarios digitales reproduce el control que ejerce el docente sobre las actividades del estudiante, incluyendo la asistencia. Esta forma de reproducción de los escenarios tradicionales puede provocar el rechazo de los estudiantes a las tecnologías 
digitales dentro de las aulas, o bien limitar su participación a lo mínimo indispensable para aprobar.

Si bien se comprende que los espacios áulicos son controlados, también se tiene la oportunidad de brindar a los estudiantes autocontrol, que debe ser enseñado paulatinamente. En este sentido, los docentes observados desperdician la oportunidad de acrecentar las habilidades de autogestión.

\subsection{Estudiantes como participantes}

Los estudiantes son jóvenes que oscilan entre los veinte y veinticinco años. En el caso de la Universidad Anáhuac Querétaro estudiaban en segundo semestre de las carreras del área de negocios y cuarto semestre de la licenciatura en Dirección de Empresas del Entretenimiento.

Los grupos observados, independientemente del nivel socioeconómico de procedencia, poseen dispositivos digitales y acceso a internet, ya sea a través de wi-fi de sus instituciones educativas o a través del uso de datos.

Todos poseían habilidades digitales básicas como realizar búsquedas, subir archivos, publicar en distintas plataformas; además algunos de ellos utilizaban los dispositivos para otro tipo de actividades, como ver series en Netflix, jugar o revisar sus redes sociodigitales para cuestiones personales.

Las características observadas en estos jóvenes corroboran las planteadas por Tapscott (2009) y Prensky (2007) con respecto a sus preferencias por la personalización, su gusto por la colaboración, la velocidad de pensamiento, el procesamiento simultáneo, su gusto por estar conectados y su visión de la tecnología como aliada, poseen algunos de los rasgos ligados a los cambios actitudinales relacionados con la apropiación de las tecnologías digitales.

Los distintos grupos de jóvenes observados se han apropiado de los dispositivos digitales y de las interfases a las cuales pueden acceder. No existe brecha tecnológica con respecto al acceso, y no se observa brecha cognitiva en el uso de los dispositivos para las cuestiones académicas excepto las señaladas por los informantes docentes.

Por ejemplo señalan que a pesar de que la mayoría de los jóvenes utilizan los dispositivos y han tomado clases de computación, no es sinónimo de que se hayan apropiado de las tecnologías para su uso escolar más allá del manejo de la paquetería de ofimática. Es importante tomar en cuenta la observación de este informante, sugiere ser flexibles y ayudar a los estudiantes a trasladar los conocimientos y habilidades digitales adquiridos en otros contextos para aplicarlos en el aula, y en caso necesario, enseñarles a utilizar la aplicación elegida por el docente.

\subsection{Los docentes como diseñadores de la participación y su concepción del aprendizaje}

Como se ha dicho, son los docentes quienes, en su papel de diseñadores de los procesos de enseñanza-aprendizaje, proponen las metodologías educativas y eligen -en primera instancia- las herramientas digitales con las que trabajaran en sus aulas, por tanto, su visión sobre el quehacer docente, la selección de tecnologías educativas, el diseño instruccional y la relación con sus estudiantes es primordial en procesos en donde se promueve la mediación tecnológica digital como parte integral del aula. 
Por otro lado, la concepción del aprendizaje es relevante puesto que cada docente tiene un punto de vista distinto sobre cómo se enseña y se aprende y "en la medida de lo posible, trata de actuar siendo consistente con ella" (Escamilla, 2011, p. 25).

Los cuatro los informantes coinciden en ver al aprendizaje como algo personal, es una perspectiva subjetivista, es decir, que "el conocimiento (y la verdad) depende de lo que los individuos poseen a priori al momento de la percepción" (Escamilla, 2011, p. 26). También apuntan que la motivación personal es importante y ven al docente como un guía.

Esta visión personal sobre el aprendizaje ha dependido de las experiencias de vida y en algunos casos, de la formación profesional. Y es necesario mencionar que los esfuerzos de las universidades para preparar a sus docentes en modelos centrados en el aprendizaje o en el aprendiz dista de ser óptima. Son pocos los docentes que están dispuestos a ver a su estudiante como un igual y en donde la única diferencia radica en que se conoce más de un tema.

Esta epistemología del aprendizaje personal, como la nombra Escamilla (2011, p. 27) también determina la forma de dar la clase, las metodologías que el docente elige. Así, las metodologías y técnicas didáctico-pedagógicas observadas en los cuatro casos fueron: 1) cátedra con retroalimentación; 2) aprendizaje invertido; 3) trabajo en equipo; 4) casos y proyectos en diversas modalidades.

La cátedra con retroalimentación es la metodología más recurrida en distintas formas. Hay quienes explican el tema apoyándose en una presentación electrónica como Miguel o Magdita, y hay quienes platican del tema de la clase apoyándose en una libreta de notas.

Otra metodología socorrida por los informantes docentes fue el trabajo colaborativo, en equipo o grupal. En la observación se corroboró que los estudiantes trabajan en equipo en el tiempo de clase y algunos de ellos se reúnen fuera de la misma para avanzar.

Todos los informantes reportan planear su clase. Unos lo hacen a través de las planeaciones didácticas que les exige la Universidad. En la observación se corroboró que todos los informantes planean su clase y dos de ellos elaboran presentaciones en PowerPoint como apoyo.

Esa empatía que los docentes se esfuerzan en lograr se ve reflejada en la receptividad de los estudiantes. En los grupos de discusión reportaron sobre las clases "la clase es muy interactiva" (Ana Mary Anáhuac-Qro). Esta afirmación se vincula con la actitud de los docentes, la preparación y el compromiso, así como con la concepción subjetivista del aprendizaje, pues se ve reflejada en el entusiasmo que muestran los distintos grupos de estudiantes.

\subsection{Selección de tecnologías digitales. Aspectos que tomar en cuenta.}

A pesar del entusiasmo de los informantes docentes por las tecnologías digitales y sus esfuerzos por incorporarlas a sus aulas, están conscientes de que no son la solución, pero que si facilitan el aprendizaje.

De hecho, hay ocasiones en las que el intento de incorporar espacios personales a los ambientes áulicos resulta contraproducente, causa resistencia en los estudiantes porque se sienten invadidos, violentados. 
Entonces, además de los criterios de selección de tecnologías digitales debido a las prestaciones de cada aplicación o servicio, los docentes han de tomar en cuenta la receptividad de los estudiantes y las posibles resistencias ante la inclusión de redes sociodigitales en los espacios áulicos o para sustituir a las plataformas diseñadas exprofeso como plataformas gestoras de contenidos.

La selección de tecnologías digitales depende de las búsquedas de los docentes, de su capacidad de incorporar nuevas opciones y del tiempo que le dediquen a adaptar su clase o preparar materiales didácticos o de apoyo. También dependen de la gratuidad de las herramientas o servicios, puesto que en ningún caso se reportó el uso de plataformas de pago, $\mathrm{y}$ en todos los casos buscaron elementos que potenciaran el aprendizaje o la motivación de los estudiantes.

\section{Cultura participativa}

La cultura participativa como categoría es observable principalmente en los escenarios digitales; en las aulas se observó en las menciones del docente sobre aquellas acciones directamente relacionadas con ingresar a las plataformas o aplicaciones, con revisar las mismas y colaborar de algún modo, ya sea publicando material propio o elaborado por otros, o bien llamados al trabajo colaborativo, en particular en los espacios digitales.

Como ya se mencionó, se tomaron en cuenta los tipos o formas de participación enunciados por Jenkins (2009): afiliación, circulación, expresión y trabajo colaborativo. Jenkins no prioriza ningún tipo por sobre otro, sin embargo, responden a un orden ascendente de involucramiento en los espacios digitales. Así, la afiliación responde a una lógica de acceso; la circulación a una lógica de reproducción, puesto que se comparte contenido producido por otros; la expresión apela a una lógica de producción; y el trabajo colaborativo responde a una lógica de cooperación, que normalmente se tiene una vez que se ha participado de las formas anteriormente enunciadas.

Si bien ya se ha dado cuenta de lo que sucedió en los espacios digitales y la forma en que estos se utilizaron, en este apartado se retoman algunos de estos aspectos para determinar qué tipo de cultura participativa existe en las aulas observadas.

\subsection{Afiliación}

Para estar afiliado, es indispensable contar con artefactos y conexión. Por tanto, para observar si existe afiliación, se tomó en cuenta el acceso a los dispositivos e internet. En todas las aulas observadas -siete aulas de cuatro informantes docentes-se encontró la presencia de laptops, teléfonos inteligentes y tabletas conectados a internet. Las cuatro instituciones educativas brindan a su comunidad conexión wi-fi que no siempre son suficiente para las necesidades del estudiantado, por lo que, si el servicio era lento o estaba saturado, tanto docentes como estudiantes hacían uso de sus datos.

Si bien en México todavía existe brecha digital, esta disminuye en el segmento observado y fue una de las razones de elegir a las aulas universitarias como escenarios de observación. Aún falta infraestructura o bien es insuficiente, pero la conectividad existe.

Como se menciona en el apartado referente los dispositivos, en todos los escenarios de observación los estudiantes llevaban dispositivos digitales, y en algunos casos más de un dispositivo por persona; los más frecuentes eran teléfonos inteligentes y laptops, que 
normalmente desplegaban en su espacio de trabajo. También se reporta la presencia de más de un dispositivo móvil por estudiante en las cuatro instituciones, sin importar el nivel económico de los estudiantes.

Todos los estudiantes tenían teléfonos inteligentes, aunque no todos los sacaran en el salón de clase. Esto puede afirmarse porque se observó que algunos los guardaban en las mochilas, por las conversaciones o porque al finalizar la clase, lo tomaban de la bolsa de mano o el bolsillo del pantalón.

Estudiantes y docentes participan a través de la afiliación, puesto que tienen usuarios en los distintos servicios, plataformas y aplicaciones en las que se trabaja o comparte información. Se encontró que los participantes se encontraban afiliados principalmente a una red social, a un servicio o mashup y a dos LMS.

\subsection{Circulación}

La observación de los escenarios digitales arroja que son los docentes quienes más circulan información a través de compartir vínculos, videos, libros, presentaciones en Prezi y otros recursos.

En los grupos de Facebook se encontró que el docente compartió con sus estudiantes videos; en uno de ellos -el de cálculo- los videos eran apoyo para explicar los temas y si bien el docente (Miguel) fue quien inició la dinámica, los estudiantes continuaron publicando videos gracias a la estrategia del docente de subir puntos por esta acción. La participación fue impulsada, como en muchos de los casos en las aulas, por una motivación extrínseca. En el otro grupo -creación y producción musical- la circulación fue mayor. El docente compartía videos para analizar y los estudiantes comentaban, respondían a sus cuestionamientos e iniciaban un diálogo entre ellos. Los estudiantes también contribuyeron con videos y fueron mucho más participativos en términos del diálogo entablado. Esto quizá se debe al tipo de asignatura, que permitió a los estudiantes un mayor engagment con los contenidos y a las características del grupo: dos estudiantes comprometidas, extrovertidas y deseosas de participar. Esta plataforma es la que más facilidades brinda para la circulación.

Los estudiantes circularon información en GoogleDrive. Esta plataforma se utilizó para compartir vínculos, libros y documentos con los estudiantes.

Como estos dos escenarios digitales servían como espacios áulicos ampliados, fueron los docentes los encargados de llevar la pauta de participación, y los estudiantes tuvieron una mínima contribución a la circulación.

No pudieron observarse las publicaciones de los estudiantes en las redes sociodigitales fuera del ámbito de los grupos creados para las asignaturas, pero su comportamiento y respuestas en los grupos de discusión apuntan a que con sus grupos de amistades comparten contenidos de acuerdo con sus intereses.

\subsection{Expresión}

La expresión o creación fue el tipo de participación más recurrente en los docentes, puesto que todos ellos elaboraron materiales de apoyo para sus asignaturas y lo compartieron en Schoology, Edmodo, Facebook y GoogleDrive. 
El tipo de material que más se produjo fueron las presentaciones electrónicas. En algunos casos los docentes compartían las presentaciones que utilizar en las clases para explicar los temas.

En el caso de los estudiantes se consideró expresión los trabajos que estaban en las distintas plataformas y servicios, como los podcasts, los mapas mentales, las presentaciones en Prezi, los avances de los protocolos de investigación, los cómics. En el caso del docenteinformante de la Anáhuac Querétaro, sus estudiantes utilizaron los documentos de GoogleDrive como cuadernos de trabajo en donde el profesor hacía observaciones mientras avanzaban. Como se reportó, el servicio también fue utilizado como reservorio de trabajos de los estudiantes.

\subsection{Colaboración}

Los cuatro docentes informantes diseñaron actividades en equipo, y en muchos de los casos los trabajos que se encontraban en las plataformas eran colectivos. Sin embargo, se observó que la falta de información a los estudiantes sobre las características del trabajo colaborativo, puesto que lo que hacían era dividir tareas y sólo integraban uno o dos miembros del equipo. En donde fue más obvio fue en la Universidad del Valle de MéxicoCampus Querétaro, puesto que en las sesiones observadas trabajaban en clase acomodando los mesa-bancos en círculos y uno o dos eran los que llevaban el trabajo y otros aportaban, incluso había los que sólo observaban o se distraían con algún juego en los teléfonos inteligentes.

Los estudiantes se organizan en grupos en donde no está el profesor, ya sea en Whatsapp o en Facebook, y poseen un carácter organizativo.

Si en esos grupos está el docente, entonces los utiliza para publicar avisos sobre la asignatura, faltas o cambios de clase.

Un hallazgo interesante fue el grupo de WhatsApp del grupo de Cálculo de la Anáhuac Querétaro, puesto que lo conformaron en la semana de exámenes, y lo utilizaban para estudiar en grupo, es decir, compartían fotografías de los ejercicios del problemario resueltos, o con explicaciones de voz; incluso hubo algún estudiante que grababa un video resolviendo un ejercicio en voz alta y lo compartía con el grupo. Este tipo de acciones son símiles a estudiar en grupo y representan un trabajo colaborativo auto-organizado (es decir, sin la intervención de ningún docente).

\section{Discusión}

A partir del análisis de las distintas categorías, puede afirmarse que en las aulas observadas existe una cultura participativa que recorre los distintos tipos enlistados por Jenkins (2009), y que, dado su carácter académico, lleva aunada la gestión académicoadministrativa tanto en las plataformas LMS como en las redes sociodigitales.

La cultura participativa en las aulas corresponde a los niveles de participación planteados por Ferrés (2011), dado que se observó juego de inferencias, participación emocional, participación mediante la interactividad tecnológica, mediante la relación, la participación en la construcción de la inteligencia colectiva y un incipiente uso de la participación para la transformación del entorno a través del trabajo en equipo a través de la organización autogestiva por parte de los estudiantes. 
Dicha cultura participativa se gestiona a partir del diseño de las asignaturas por parte de los docentes, quienes conciben a las tecnologías digitales como aliadas, como parte de la vida cotidiana -propia y de los estudiantes- y buscan crear experiencias de aprendizaje que motiven a sus estudiantes a través de la empatía y entendimiento del uso transparente de las tecnologías digitales.

Es necesario hacer énfasis en la capacitación docente en temáticas de apropiación de tecnología digital y en las metodologías de clases mediadas, porque no todos los docentes poseen la iniciativa, el tiempo o el gusto por las tecnologías, por lo que el desconocimiento y los prejuicios sobre las redes sociodigitales - por citar el ejemplo más común- tiene como consecuencia que sean pocos los profesores que aprovechen las posibilidades educativas de dichas tecnologías.

Los informantes docentes poseen en común, además de su apropiación de las tecnologías digitales como parte de la clase, una visión subjetiva del aprendizaje, la utilización de la cátedra con retroalimentación y el trabajo colaborativo en distintos formatos. Cada uno de ellos seleccionó distintas aplicaciones o servicios, sin embargo, tienen en común su uso como espacios para tareas y trabajos de sus estudiantes.

Los estudiantes poseen uno o más dispositivos para conectarse a internet y los utilizan para fines académicos dentro de las clases y para usos personales o recreativos, lo que no les impide seguir con el trabajo de clase, en especial si es en equipo, puesto que cada grupo gestiona el desempeño de sus miembros. Al respecto se observó que hace falta mayor énfasis en la metodología de trabajo colaborativo para que los estudiantes logren resultados más satisfactorios.

Facebook es útil siempre y cuando el grupo esté de acuerdo en utilizarla, debido a que es un espacio personal. Permite un alto grado de circulación de contenidos y ello invita a un intercambio dialógico en espacios digitales sobre los temas de clase. Además, su diseño tecnológico permite compartir la expresión o creación de contenidos propios en distintos formatos.

GoogleDrive es un servicio que brinda la facilidad de un trabajo síncrono y asíncrono, y permite correcciones y mejoras a los trabajos antes de que sean las fechas de entrega, así como el almacenamiento de archivos diversos y su ordenamiento por carpetas. Por su naturaleza, facilita la expresión o creación de contenidos, puesto que proporciona espacios de trabajo. Este servicio es el que más facilita una cultura participativa enfocada al trabajo colaborativo.

Entonces, los elementos de la cultura de la participación que se identifican en los entornos educativos observados son:

1) Los escenarios físicos de las universidades permiten a los participantes conectarse y trabajar con las condiciones adecuadas para ello, si bien en algunos de los casos sería necesario ampliar la infraestructura tecnológica para brindar una mejor conexión debido al gran número de estudiantes que requieren del servicio.

2) Los escenarios digitales permiten a los participantes publicar, compartir, dialogar y trabajar colaborativamente a través de distintos servicios. En los casos observados se encontró predilección por una red social (Facebook), un servicio integrado o mashup (GoogleDrive) y dos plataformas de gestión del aprendizaje. Los 
escenarios fueron utilizados además como reservorios del trabajo de los estudiantes.

3) A pesar de utilizar las tecnologías digitales, los docentes informantes requieren conocer otras plataformas y herramientas puesto aunque puede utilizarse Facebook como reservorio de los materiales elaborados por los docentes, no es el mejor uso y puede causar rechazo por parte de los estudiantes. Servicios como Edmodo o Schoology son más adecuados para los intercambios académicos puesto que están diseñados para eso.

4) Existe una interrelación entre tres actantes: dispositivos, docentes y estudiantes. Los dispositivos forman parte de la triada dado que son los que permiten acceder a los escenarios digitales; docentes y estudiantes son quienes interactúan en las aulas $\mathrm{y}$ en los escenarios digitales a través de diferentes tipos de participación, enriqueciendo los contenidos académicos o bien a través de los trabajos, conversaciones, comentarios y diálogos en privado.

5) La cultura participativa se manifiesta a través de la afiliación, la circulación, la expresión y el trabajo colaborativo en distintos niveles, dependiendo del grupo y del docente, así como de los escenarios digitales en los que se lleve a cabo el intercambio. Existen escenarios, como las redes sociodigitales que fomentan la circulación, y al tratarse de ámbitos académicos, la creación se ve mayormente reflejada en los trabajos de los estudiantes y los materiales educativo que el docente comparte.

6) Los docentes que participaron en este estudio poseen en común entusiasmo por las tecnologías digitales y un uso transparente de las mismas, lo cual comparten con sus estudiantes a través del uso de dispositivos y escenarios digitales. También tienen un punto de vista subjetivo del aprendizaje y la utilización intuida del conectivismo y de las teorías cognitivo-constructivistas, lo que se refleja en el diseño de actividades, el tipo de cátedra de hacen y la relación que tienen con sus estudiantes.

7) La selección de los escenarios digitales para usos académicos depende de la predilección del docente y de su funcionalidad para servir como reservorios, espacios de diálogo, para tener control sobre el visualizado de los materiales (por parte de los estudiantes) y otras funciones como pase de lista o trabajo colaborativo simultáneo. Otro de los requisitos mencionados es la gratuidad, que, si bien es una ventaja en términos económicos, resulta un punto de reflexión, dado que todos los servicios requieren y generan de datos que las compañías comercializan o hacen otro tipo de usos. Las tecnologías, cualesquiera que estas sean, no son inocuas.

8) Los estudiantes son jóvenes universitarios, con acceso a distintos dispositivos digitales y conexión a internet. Poseen habilidades digitales básicas y dependiendo de la asignatura en la que participen, son capaces de trabajar colaborativamente, gestionar la colaboración sin intervención del docente, de crear contenidos académicos propios a través de los trabajos y tareas, y de compartir o circular contenidos relacionados con las temáticas de clase. 


\section{Referencias bibliográficas}

Álvarez-Gayou, J.L. (2012). Cómo hacer investigación cualitativa. Fundamentos y metodología. Barcelona: Paidós.

Cobo Romaní, C. y Pardo Kuklinski, H. (2007). Planeta Web 2.0. Inteligencia colectiva o medios fastfood. Grup de Recerca d'Interaccions Digitals, Universitat de Vic. Flacso México: Barcelona / México DF.

Escamilla, J. (2011). Selección y uso de tecnología educativa. México: Trillas-ITESM.

Ferrés, J. (2011). Educomunicación y cultura participativa. En R. Aparici Marino (Coord.) Educomunicación: Más allá del 2.0 (pp. 251-266). Barcelona:Gedisa.

Fumero, A., Roca, G. y Sáez Vacas, F. (2007). Web 2.0. Fundación Orange España.

Jenkins, H. (2009). Confronting the Challenges of Participatory Culture: Media Education for the 21st Century. Cambridge, Massachusetts McArtur Fundation/The MIT Press. Recuperado de https://bit.ly/2PUOea9

Latour, B. (1996). On actor-network theory: A few clarifications. Soziale Welt, 47(4), 369381. Recuperado de https://bit.ly/2M4y7pc

Prensky, M. (2007) Digital game-based learning. New York: Mc Graw Hill.

Tapscott, D. (2009). Grown up Digital: How the Net Generation Is Changing the World. New York: McGraw Hill.

Wikipedia (2017). Google Drive. Recuperado de https://bit.ly/2EppD84 